\title{
Impaired wheel running exercise in CLC-1 chloride channel-deficient myotonic mice
}

\author{
Erik van Lunteren ${ }^{1,2}{ }^{*}$, Michelle Moyer $^{1}$, Jessica Cooperrider ${ }^{1}$ and Jennifer Pollarine ${ }^{1}$ \\ ' Cleveland Department of Veterans Affairs Medical Center, Cleveland, OH, USA \\ 2 Pulmonary and Critical Care Division, Department of Medicine, Case Western Reserve University, Cleveland, OH, USA
}

Edited by:

Philip S. Clifford, Medical College of

Wisconsin, USA

Reviewed by:

Leonardo F. Ferreira, University of

Florida, USA

Robert Hester, University of

Mississippi, USA

${ }^{*}$ Correspondence:

Erik van Lunteren, Cleveland

Department of Veterans Affairs

Medical Center, Pulmonary 111 J(W),

10701 East Boulevard, Cleveland,

$\mathrm{OH}$ 44106, USA.

e-mail:exv4@cwru.edu

\begin{abstract}
Background: Genetic deficiency of the muscle CLC-1 chloride channel leads to myotonia, which is manifested most prominently by slowing of muscle relaxation. Humans experience this as muscle stiffness upon initiation of contraction, although this can be overcome with repeated efforts (the "warm-up" phenomenon). The extent to which CLC-1 deficiency impairs exercise activity is controversial. We hypothesized that skeletal muscle CLC-1 chloride channel deficiency leads to severe reductions in spontaneous exercise. Methodology/Principal Findings: To examine this quantitatively, myotonic CLC- 1 deficient mice were provided access to running wheels, and their spontaneous running activity was quantified subsequently. Differences between myotonic and normal mice in running were not present soon after introduction to the running wheels, but were fully established during week 2 . During the eighth week, myotonic mice were running significantly less than normal mice $(322 \pm 177$ vs $5058 \pm 1253 \mathrm{~m} /$ day, $P=0.025)$. Furthermore, there were considerable reductions in consecutive running times $(18.8 \pm 1.5 \mathrm{vs} 59.0 \pm 3.7 \mathrm{~min}, P<0.001)$ and in the distance per consecutive running period ( $58 \pm 38$ vs $601 \pm 174 \mathrm{~m}, P=0.048$ ) in myotonic compared with normal animals. Conclusion/Significance: These findings indicate that CLC-1 chloride deficient myotonia in mice markedly impairs spontaneous exercise activity, with reductions in both total distance and consecutive running times.
\end{abstract}

Keywords: myotonia congenita, exercise, genetic, CLC-1 chloride channel, skeletal muscle

\section{INTRODUCTION}

There is a diversity of chloride channels in mammalian tissues, including (a) CLC chloride channels, which are often voltage gated, (b) $\mathrm{GABA}_{\mathrm{A}}$ and glycine receptors, which are ligand gated, and (c) the cystic fibrosis transmembrane conductance regulator. The CLC chloride channel family in turn consists of many subtypes, including CLC-1 through CLC-7 plus CLC-Ka and CLC-Kb. These channels differ in cellular location, with CLC-1, CLC-2, CLC-Ka, and $\mathrm{CLC}-\mathrm{Kb}$ residing on the plasma membrane, and the others on intracellular membranes. Genetic defects in these channels cause a number of human diseases, including myotonia congenita, Bartter syndrome III, Dent's disease, osteopetrosis, and possibly seizures (Jentsch et al., 2005).

CLC-1 is found predominantly in skeletal muscle (Steinmeyer et al., 1991a). Myotonia congenita in humans results from over 80 different mutations in the CLCN1 gene on chromosome 7. Two clinical forms are recognized, autosomal dominant Thomsen's and autosomal recessive Becker's myotonia congenita (Koch et al., 1992; Ptacek et al., 1993; Kleopa and Barchi, 2002; Pusch, 2002; Jentsch et al., 2005; Puljak and Kilic, 2006). Mutations of this gene with impaired CLC-1 chloride channel function are also found in goats and mice (Heller et al., 1982; Watkins and Watts, 1984; Mehrke et al., 1988; Reininghaus et al., 1988; Steinmeyer et al., 1991b; Gurnett et al., 1995; Beck et al., 1996; Jentsch et al., 2005). Reduced chloride channel conductance leads to membrane hyperexcitability, which in turn leads to repetitive electrical discharges following muscle activation. The net result is a slowing of muscle relaxation as a result of the continued electrical activity (Entrikin et al., 1987; Reininghaus et al., 1988). The myotonia diminishes with repeated muscle contractions, but returns quickly following a period of inactivity (Entrikin et al., 1987; van Lunteren et al., 2004). In humans this is experienced as increased muscle stiffness which interferes with exertion. Goats with myotonia develop intermittent muscle stiffening causing them to fall over especially after being startled, whereas mice demonstrate a prolonged stiff leg extensor posture after shaking the cage or dropping the animals from a small height. Some but not all studies have also reported weakness in the intact animal as well as impaired muscle contractile performance when studied experimentally (Heller et al., 1982; Watkins and Watts, 1984; Entrikin et al., 1987; Reininghaus et al., 1988; van Lunteren et al., 2007a,b).

The extent to which CLC-1 chloride channel deficiency affects exercise performance is controversial. Descriptions of human disease typically mention impairment of exercise performance, in particular at or near the onset of exercise, although there are also reports of subjects who engage in vigorous exercise (Entrikin et al., 1987; Burnham, 1997; Weinberg et al., 1999; Stimson, 2001; Colding-Jorgensen, 2005) - however controlled quantitative data are scant. The original report of myotonic mice notes that the animals walk almost normally, albeit more slowly and slightly stiffly, and are able to swim albeit with some degree of hindleg uncoordination; only when placed in an ice-water bath does the 
impairment in swimming become prominent, although quantitative data are lacking (Heller et al., 1982). In contrast, there is one quantitative study which documented a very large (60-65\%) reduction in spontaneous locomotor activity of myotonic mice while in a cage (Heimann et al., 1988). The latter data, however, most likely reflects predominantly low distance walking rather than moderate to high distance walking and running. Based on the above information, it is unclear whether CLC-1 deficiency has mild or severe adverse effects on exercise activity. The purpose of the present study was to test the hypothesis that skeletal muscle CLC-1 chloride channel deficiency leads to severe impairments in spontaneous exercise, which was tested in myotonic mice by assessing voluntary wheel running. Voluntary wheel running was chosen as the model for a moderate to high amount of exercise because normal rodents typically spontaneously walk and run $\sim 4-10 \mathrm{~km} /$ day when provided access to running wheels (DupontVersteegden et al., 1994; Carter et al., 1995; Hayes and Williams, 1996; van Lunteren and Moyer, 2003; van Lunteren et al., 2004). Furthermore, as noted previously, descriptions of the manifestations of the myotonia differ among humans, goats, and mice. In contrast, voluntary walking and running is a form of exercise that is common to both mice and humans, so that the findings in myotonic mice will be directly applicable to humans with myotonia congenita.

\section{MATERIALS AND METHODS}

All studies were approved by the Institutional Animal Care and Use Committee at the Cleveland Department of Veterans Affairs Medical Center (Cleveland, OH, USA), and performed in accordance with NIH animal care guidelines. Male homozygous (-/-) myotonic mice (SWR/J-Clcn $1^{\text {adr/mto } / J ;-/-; ~} n=3$ ) and phenotypically normal control animals $( \pm$ or $+/+; n=4)$ were obtained from Jackson Laboratories (Bar Harbor, ME, USA). Throughout their lifetime heterozygous $( \pm)$ mice are behaviorally indistinguishable from homozygous $(+/+)$ normal controls; furthermore, the electrical activity of heterozygotes can not be distinguished from homozygous normal controls (Heller et al., 1982). Thus many studies of myotonic mice group heterozygous $( \pm)$ and homozygous $(+/+)$ mice together as controls (Heller et al., 1982; Fuchtbauer et al., 1988; Mehrke et al., 1988; Goblet and Whalen, 1995; Kramer et al., 1998; van Lunteren et al., 2007a,b), similar to the present study. At the time of entry into the study the animals were 5-6 weeks old. Animals were kept in a 12:12 light:dark cycle. Assessment of spontaneous running was done using standard size polycarbonate living chambers which contained running wheels (van Lunteren and Moyer, 2003; van Lunteren et al., 2004). The running wheels were equipped with a magnetic counter, the output of which was sent to a PC-type computer (Lafayette Instruments, Lafayette, IN, USA). This allowed quantification of the number of revolutions, and multiplying this by the circumference of the running wheels provided measurements of distance. The animals spent 8 weeks in running wheelequipped housing, and were given ample access to food and water $24 \mathrm{~h}$ /day. All animals were housed singly while their running was assessed.

Recordings of the number of rotations of each running wheel were performed $24 \mathrm{~h} /$ day for the entire 8 week period. The total distance each animal ran per day was initially calculated. The approach used in previous studies (Hara et al., 2002; van Lunteren et al., 2004) of identifying active running times was adopted; specifically any $10 \mathrm{~min}$ interval during which the animals ran enough to produce at least one rotation of the wheel was defined as an active running period. Higher thresholds for defining active running times were also examined (see Results). Average consecutive running times were calculated from the number of 10 min periods in a row during which the animals ran. Thus the consecutive running time indicates the total duration of time during which the animals ran enough to produce at least the pre-defined minimum number of wheel rotations during each $10 \mathrm{~min}$ interval, and does not imply that the animals ran without stopping the entire time (Hara et al., 2002; van Lunteren et al., 2004). In previous studies of normal rats we found that when animals are provided access to a running wheel, they gradually increase their running distance over the course of 3-5 weeks and then remained relatively stable thereafter (van Lunteren and Moyer, 2003; van Lunteren et al., 2004). Statistical analysis was performed with the unpaired $t$-test for comparisons of two data sets. Analysis of variance, followed by the Student-Newman-Keuls test in the event of a significant analysis of variance result, was used for statistical testing of multiple data sets. A $P$ value of $<0.05$ (two-tailed) was chosen to indicate significance.

\section{RESULTS}

The myotonic mice used for the running studies weighed considerably less than the wild type mice ( $12.7 \pm 0.3$ vs $25.5 \pm 0.3 \mathrm{~g}$ ), as has also been noted in previous studies of this mouse model (Heller et al., 1982; Heimann et al., 1988; van Lunteren et al., 2007a). Delayed muscle relaxation during isometric contractions was verified in previous muscle contractile studies of CLC-1 chloride channel-deficient myotonic animals (Heller et al., 1982; Heimann et al., 1988; Mehrke et al., 1988; Reininghaus et al., 1988; van Lunteren et al., 2004, 2007b).

\section{RUNNING ACTIVITY AT 8 WEEKS}

Examples of the temporal pattern of running over the course of each day during the eighth week are depicted in Figure 1. Both normal and myotonic mice did the vast majority of their wheel running between $8 \mathrm{pm}$ and $8 \mathrm{am}$, as expected for nocturnal animals. Normal mice almost always ran for multiple consecutive 10-min periods, typically 15-20 consecutive periods at the onset of the night, and then fewer consecutive periods as the night went on. In contrast, myotonic mice only ran only a few times per night, and for short periods of time per episode (typically no more than three consecutive 10-min periods).

Data were obtained and averaged for all 7 days of the eighth week of running (Figure 2). Normal mice ran significantly further per day than myotonic mice (Figure 2A). In addition, all normal animals averaged more than $1300 \mathrm{~m} /$ day (with most running in excess of $5000 \mathrm{~m} /$ day), whereas all myotonic mice averaged less than $700 \mathrm{~m} /$ day. The mean consecutive running time was significantly shorter for myotonic than normal mice (Figure 2B). Changing the threshold for defining the consecutive running time from at least one revolution to a higher value did not change 


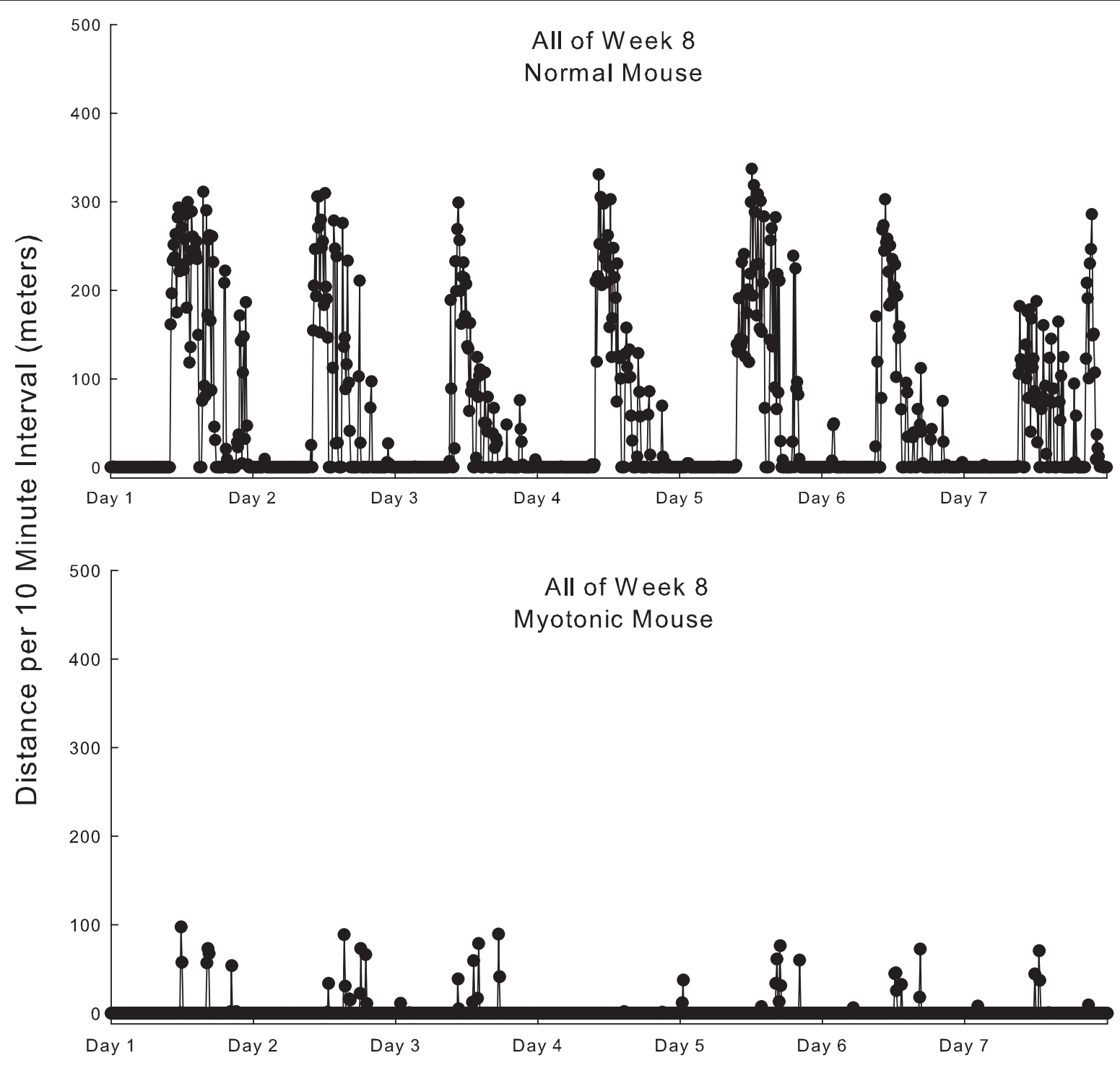

FIGURE 1 | Example of running behavior of a normal and myotonic mouse during all of week 8. The distance run is quantified for each 10 min interval as a function of time during the day. Each day depicted is from 8 am one morning until 8 am the following morning.

the finding of shorter consecutive running times in myotonic compared with control animals, as depicted in Figure 2C for a threshold of $3 \mathrm{~m} / 10 \mathrm{~min}$ interval. Similarly, the average consecutive running distance (defined as the total distance run during each consecutive running period) was lower for myotonic than control mice (Figure 2D).

\section{TIME COURSE OVER WHICH CHANGES IN RUNNING OCCURRED}

The time course of changes in running distance of normal and myotonic mice over the 8-week period is depicted in Figure 3A. During the first few days of access to the running wheels, the normal mice gradually increased the amount of running. At 2 weeks the animals averaged $>4 \mathrm{~km} /$ day, and this distance was maintained thereafter (albeit with a slight downward trend). In contrast, the myotonic mice did not increase the running distance from the first few days to later time periods. Running distances were significantly greater for normal than myotonic mice at 2,4 , and 8 weeks.

The above temporal patterns seen with total running distance were also noted for consecutive running time (Figure 3B). Myotonic mice had significantly lower consecutive running times than normal mice at 2, 4, and 8 weeks, but not at 2-3 days.

\section{DISCUSSION}

The present study found substantial reductions in spontaneous exercise activity of myotonic mice. Not only was the total distance run per day reduced substantially, but the animals ran for shorter consecutive durations, and for shorter distances each time they ran. Furthermore, normal mice gradually increased the running distance and consecutive running times when first provided access to the running wheels, whereas myotonic mice did not. 


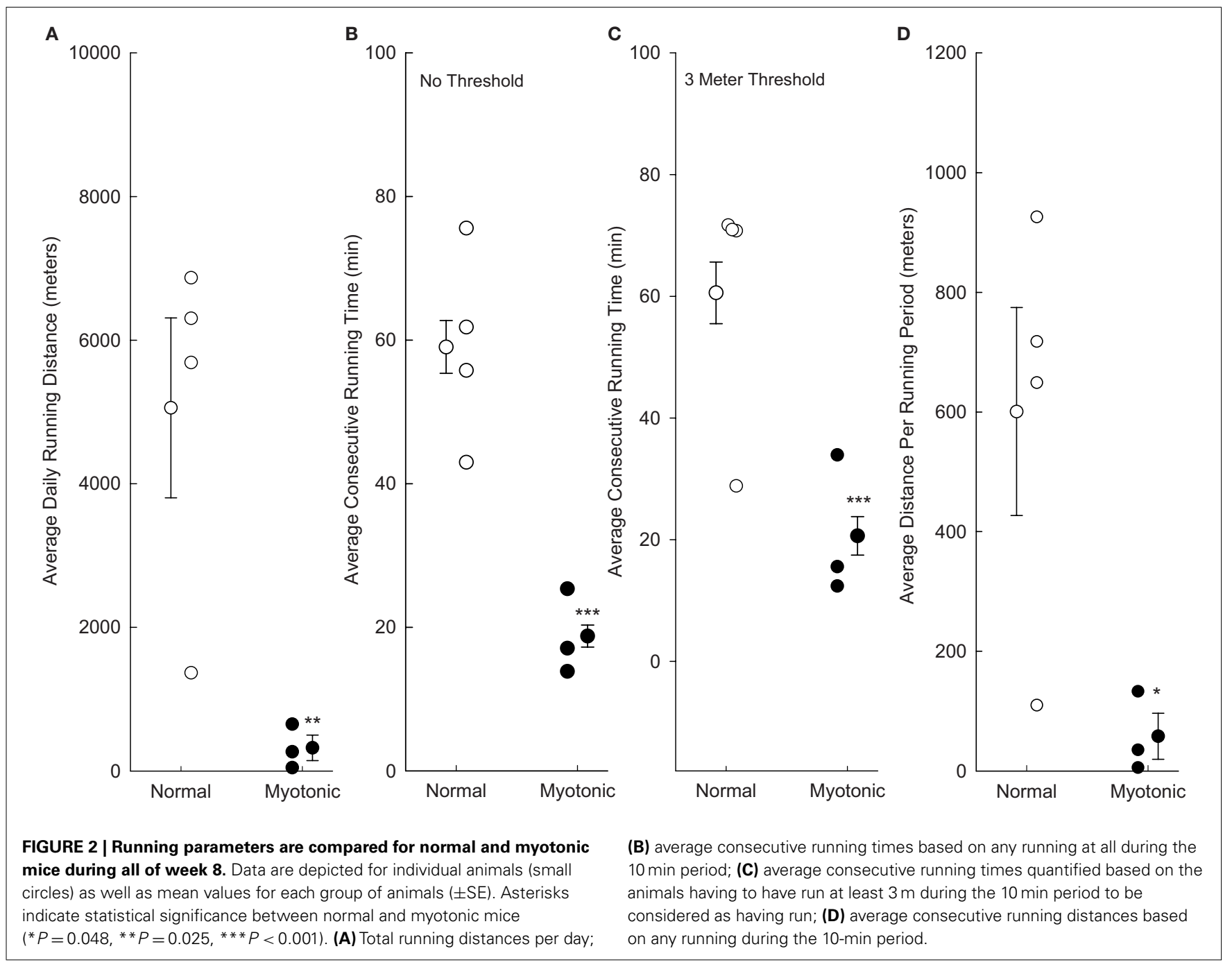

One issue about the design of the present study is the use of relatively small sample sizes of three myotonic mice and four normal mice. Large differences in running distances and times were noted between myotonic and normal animals which greatly exceeded variability in values within each group, resulting in statistically significant findings. Calculations were performed on the 8-week data to determine whether the study was adequately powered to have at least an $80 \%$ chance of detecting significant differences at a $P<0.05$ level. The following power values were found: $96 \%$ for average running distance, $100 \%$ for average consecutive running time with no threshold, $89 \%$ for average consecutive running time with a $3-\mathrm{m}$ threshold, and $86 \%$ for average distance per running period. Thus power analysis indicates that the sample sizes were indeed adequate to statistically detect the large differences in running parameters between myotonic and control mice.

Many descriptions of exercise capacity in humans with myotonia congenita are qualitative rather than quantitative (Ptacek et al., 1993; Burnham, 1997; Weinberg et al., 1999; Stimson, 2001; Colding-Jorgensen, 2005) or compare myotonic subjects before and after an intervention rather than myotonic vs normal subjects (Birnberger et al., 1975; Hammaren et al., 2005). Impaired ability to perform athletic activities is described frequently, in particular for strenuous activities. On the other hand, various degrees of impairment can be overcome by engaging in a suitable antecedent warm-up period, and furthermore the time course over which myotonia dissipates is considerably faster than the time course over which fatigue develops during high intensity repetitive contractions (van Lunteren et al., 2011). As a result, some subjects with myotonia congenita are able to engage successfully (albeit not always fully normally) in strenuous athletic activities (Burnham, 1997; Weinberg et al., 1999; Stimson, 2001; Colding-Jorgensen, 2005).

Heller et al. (1982) noted that the animals walk almost normally, but on closer inspection their gait is slightly "stiff" and walking is slower than unaffected littermates. Subsequently Entrikin et al. (1987) described a stiffed-legged hindlimb gait. Heimann et al. (1988) video-recorded mice while on a plane surface, and found that the relative reduction in spontaneous activity of myotonic mice (60-65\%) was greater than the magnitude of 


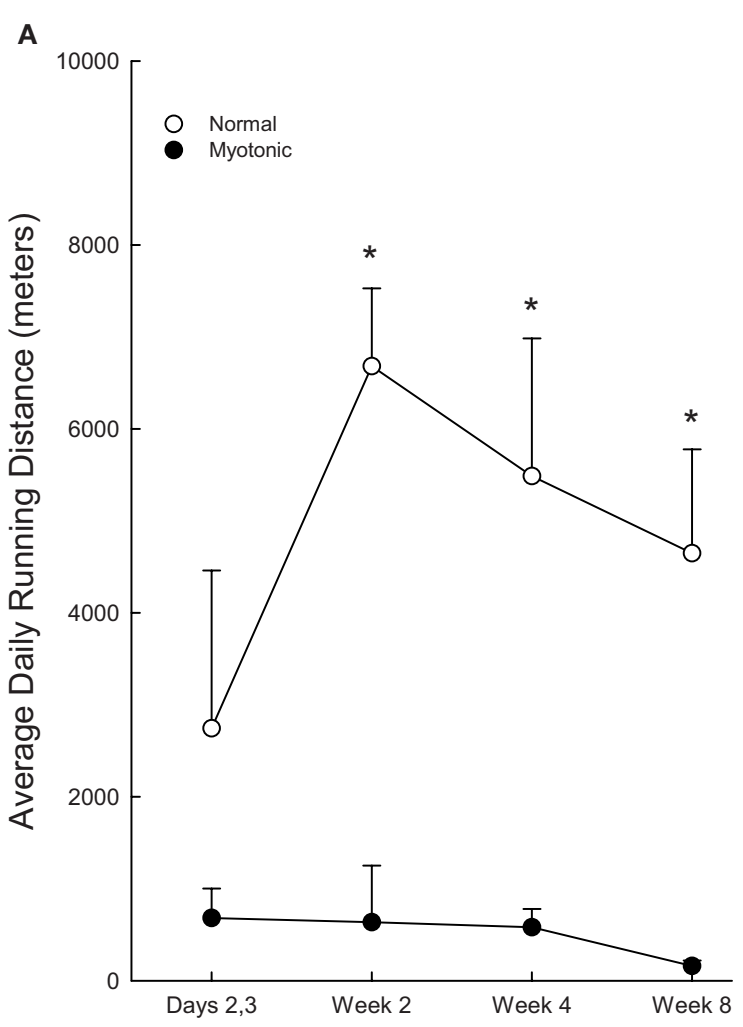

FIGURE 3 | Changes in average daily running distances (A) and average consecutive running times (B) for normal and myotonic mice as a function of time after being provided access to the running wheels.

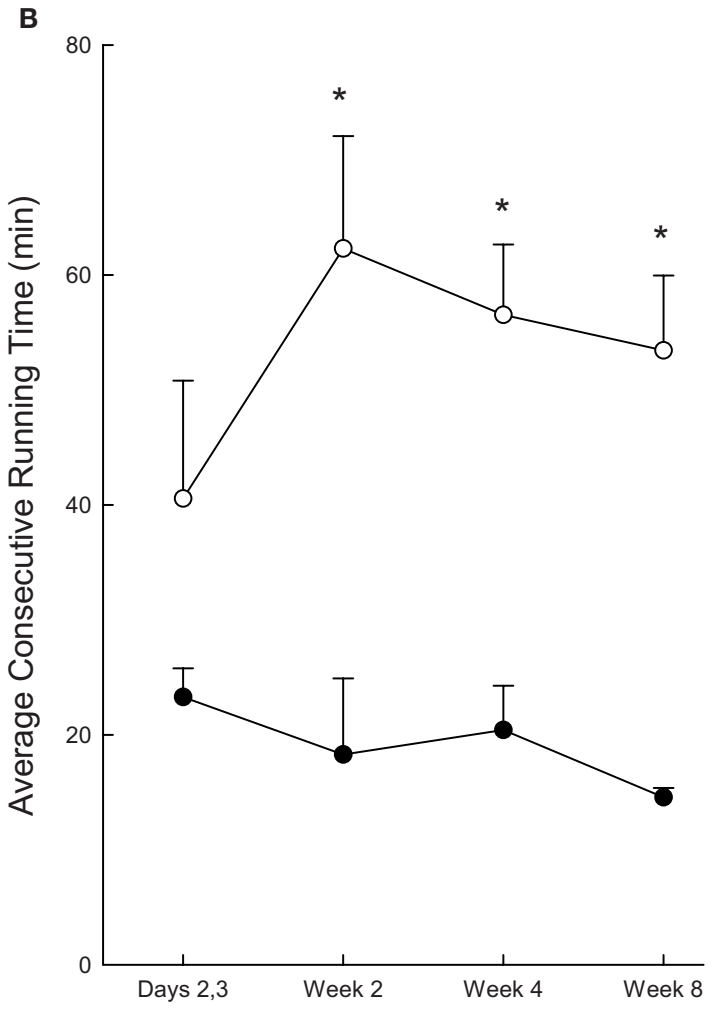

The 2, 4, and 8-week data were quantified during the last 2 days of the week. Asterisks $\left(^{*}\right)$ indicate significant differences between normal and myotonic mice. reduced activity (50-55\%) seen in dystrophin-deficient $m d x$ dystrophic mice (an animal model of human Duchenne muscular dystrophy).

Although the running activity of myotonic mice has not been reported previously, that of dystrophin-deficient $m d x$ dystrophic mice has been quantified. Dupont-Versteegden et al. (1994) found that control mice ran $\sim 7 \mathrm{~km} /$ day whereas $m d x$ dystrophic mice ran $\sim 5 \mathrm{~km} /$ day. Carter et al. (1995) found no significant running deficit in the young (4 week old) dystrophic mice but a $50-70 \%$ reduced distance run in adult (6 month old) dystrophic mice. Hayes and Williams (1996) found average running distances of $30 \mathrm{~km} /$ week in mdx dystrophic mice and $45 \mathrm{~km} /$ week in control mice. The present study found that myotonic mice had deficits in running distance of $\sim 85-90 \%$, thus exceeding the above reported reductions seen in $m d x$ mice. Hayes and Williams (1996) also reported on the running behavior of $\alpha 2$-laminindeficient $d y / d y$ dystrophic mice (an animal model of human classic congenital muscular dystrophy). The $d y / d y$ mice have greater generalized weakness than $m d x$ mice (Carter et al., 1995; Jentsch et al., 2005), and this was reflected in very large impairments of running distance (80-95\%). Therefore the running activity deficit of myotonic mice is comparable to that of $d y / d y$ dystrophic mice.

Hara et al. (2002) found shortened continuous running times in the $m d x$ dystrophic compared with control animals (20-30 vs
50-80 min, depending on age). A running endurance abnormality also occurs in streptozotocin-induced diabetes, with average values at 8 weeks of 16 and $40 \mathrm{~min}$ in diabetic and normal rats, respectively (van Lunteren et al., 2004). In the present study, the deficit in the average consecutive running time of the myotonic animals was in the 60-70\% range, and thus comparable in magnitude to the deficits seen in both $m d x$ dystrophic mice and diabetic rats.

Several mechanisms may contribute to the impaired running activity (present study) and activity levels (Heimann et al., 1988) of the myotonic mice. First, the myotonic mice were smaller than the normal mice despite comparable ages, in agreement with previous reports of this mouse model (Heller et al., 1982; Heimann et al., 1988; van Lunteren et al., 2007a). Reduced weight due to fat loss should improve running, whereas reduced weight due to sarcopenia should reduce running. Second, it is possible that the animals did not want to run instead of that they could not run, similar to what has been reported with hormonal interventions. Steinmeyer et al. (1991a) examined the tissue distribution of CLC1 by northern blot analysis. There was a prominent band in skeletal muscle, but faint bands in kidney, liver, heart, and a smooth muscle cell line. Thus CLC-1 is found predominantly, but probably not exclusively, in skeletal muscle making it relatively unlikely that it could affect a mouse's motivation to run. Third, the myotonic mice have substantial electrical and mechanical myotonia as a direct 
effect of the CLC-1 chloride channel loss, as reported previously (Heller et al., 1982; Heimann et al., 1988; Mehrke et al., 1988; Reininghaus et al., 1988; van Lunteren et al., 2004, 2007b), and this certainly will impair muscle performance during intermittent contractions. Fourth, muscle from humans and animals with myotonia congenita has structural, biochemical, and contractile alterations which go beyond the direct effects of the markedly reduced or absent CLC-1 channels. This includes alterations in fiber type composition and myosin isoform distribution (in particular loss of type IIB fibers and myosin), down regulation of parvalbumin, and abnormal expression of myogenic regulatory factors (MyoD and myogenin; Stuhlfauth et al., 1984; Reininghaus et al., 1988; Agbulut et al., 2004). In addition, variable degrees of weakness have been noted in the intact animal (Heller et al., 1982; Watkins and Watts, 1984), and studies of isolated muscles have noted impaired performance during the contraction phase of the contraction-relaxation cycle under both isometric and isotonic conditions (Entrikin et al., 1987; Reininghaus et al., 1988; van Lunteren et al., 2007a,b). The extent of impairment is considerable, for example isometric force is reduced by $35-65 \%$ whereas isotonic power and work impairments may exceed $50 \%$ (van Lunteren et al., 2007a,b). Fifth, there may have been a training response during the course of the 8-weeks of running in the normal mice which exceeded that of the myotonic mice. However, both groups of mice were housed in conventional cages prior to the onset of the 8 -week running period, and therefore the early difference between groups can not be attributed to training responses. Finally, it is unlikely that pain contributed to differences between groups, as there was no evidence for the myotonic mice having pain while running, and we are not aware of pain being a significant issue in this disease.

It is interesting to consider whether exercise training might revert the effects of the CLC- 1 chloride channel deficiency. The present data would suggest that little or no mitigation occurs.

\section{REFERENCES}

Agbulut, O., Noirez, P., Butler-Browne, G., and Jockusch, H. (2004). Specific isomyosin proportions in hyperexcitable and physiologically denervated mouse muscle. FEBS Lett. 561, 191-194.

Beck, C. L., Fahlke, C., and George, A. L. (1996). Molecular basis for decreased muscle chloride conductance in the myotonic goat. Proc. Natl. Acad. Sci. U.S.A. 93, 11248-11252.

Birnberger, K. L., Rudel, R., and Struppler, A. (1975). Clinical and electrophysiological observations in patients with myotonic muscle disease and the therapeutic effect of N-propyl-ajmalin. J. Neurol. 210, 99-110.

Burnham, R. (1997). Unusual causes of stiffness in two hockey players. Clin. J. Sport Med. 7, 137-140.

Carter, G. T., Wineinger, M. A., Walsh, S. A., Horasek, S. J., Abresch, R. T., and Fowler, W. M. Jr. (1995).
Effect of voluntary wheel-running exercise on muscles of the $\mathrm{mdx}$ mouse. Neuromuscul. Disord. 5, 323-332.

Colding-Jorgensen, E. (2005). Phenotypic variability in myotonia congenita. Muscle Nerve 32, 19-34.

Dupont-Versteegden, E. E., McCarter, R. J., and Katz, M. S. (1994). Voluntary exercise decreases progression of muscular dystrophy in diaphragm of mdx mice. J. Appl. Physiol. 77, 1736-1741.

Entrikin, R. K., Abresch, R. T., Sharman, R. B., Larson, D. B., and Levine, N. A. (1987). Contractile and EMG studies of murine myotonia (mto) and muscular dystrophy (dy/dy). Muscle Nerve 10, 293-298.

Fuchtbauer, E. M., Reininghaus, J., and Jockusch, H. (1988). Developmental control of the excitability of muscle: transplantation experiments on a myotonic mouse mutant. Proc. Natl. Acad. Sci. U.S.A. 85, 3880-3884.

If there was an improvement in muscle function with repetitive running one would expect that the myotonic animals should increase the amount of running during the 8-weeks of study; rather there was actually a small decrease over time.

The present study used voluntary rather than forced exercise, and thus assessed the extent to which exercise gets performed on a regular basis rather than maximum exercise capacity. Forced exercise regimens in rodents typically involve application of noxious or painful stimuli (e.g., electrical shocks) to ensure that the animals' effort is maximal. The resultant stress may produce substantial physiological responses that differ from those of exercise itself. In contrast, most human exercise is done on a voluntary basis. At times humans participate in highly structured exercise regimens during athletic or military training, but frequently exercise performance is encouraged verbally rather than enforced by noxious or painful stimuli. Therefore, data from a myotonic rodent model of forced exercise would be less applicable to myotonic humans than a rodent model of voluntary exercise. On the other hand forced treadmill running might have allowed us to test whether a warm-up period improves exercise performance.

In conclusion, CLC-1 chloride channel-deficient myotonic mice have deficits in spontaneous exercise performance. The magnitude of the deficit is quite large, and in many respects as great as that seen in animal models of muscular dystrophy. One limitation of the present study with respect to extrapolating these findings to humans with myotonia congenita is that myotonia dissipates with repeated contractions (the "warm-up phenomenon"), and thus it may be possible for humans with CLC-1 deficient myotonia to overcome at least part of the exercise deficit by a well-designed warm-up regimen prior to initiating exercise.

\section{ACKNOWLEDGMENT}

This study was supported in part by funding from the Department of Veterans Affairs Medical Research Service.

Goblet, C., and Whalen, R. G. (1995) Modifications of gene expression in myotonic murine skeletal muscle are associated with abnormal expression of myogenic regulatory factors. Dev. Biol. 170, 262-278.

Gurnett, C. A., Kahl, S. D., Anderson, R. D., and Campbell, K. P. (1995). Absence of skeletal muscle sarcolemma chloride channel ClC-1 in myotonic mice. J. Biol. Chem. 270, 9035-9038.

Hammaren, E., Kjellby-Wendt, G., and Lindberg, C. (2005). Quantification of mobility impairment and selfassessment of stiffness in patients with myotonia congenita by the physiotherapist. Neuromuscul. Disord. 15, 610-617.

Hara, J., Nolan, P. M., Scott, M. O., Bucan, M., Wakayama, Y., and Fischbeck, K. H. (2002). Running endurance abnormality in $\mathrm{mdx}$ mice. Muscle Nerve 25, 207-211.

Hayes, A., and Williams, D. A. (1996). Beneficial effects of voluntary wheel running on the properties of dystrophic mouse muscle. J. Appl. Physiol. 80, 670-679.

Heimann, P., Augustin, M., Wieneke, S., Heising, S., and Jockusch, H. (1988). Mutual interference of myotonia and muscular dystrophy in the mouse: a study on ADR-MDX double mutants. Neuromuscul. Disord. 8 , 551-560.

Heller, A. H., Eicher, A. M., Hallett, M., and Sidman, R. L. (1982). Myotonia, a new inherited muscle disease in mice. J. Neurosci. 2, 924-933.

Jentsch, T. J., Poet, M., Fuhrmann, J. C., and Zdebik, A. A. (2005). Physiological functions of CLC Clchannels gleaned from human genetic disease and mouse models. Annu. Rev. Physiol. 67, 779-807.

Kleopa, K., and Barchi, R. L. (2002). Genetics disorders of neuromuscular ion channels. Muscle Nerve 26, 299-325. 
Koch, M. C., Steinmeyer, K., Lorenz, C., Ricker, K., Wolf, F., Otto, M., Zoll, B., Lehmann-Horn, F., Grzeschik, K., and Jentsch, T. J. (1992). The skeletal muscle chloride channel in dominant and recessive human myotonia. Science 257, 797-800.

Kramer, R., Lochmuller, H., Abicht, A., Rudel, R., and Brinkmeier, H. (1998). Myotonic ADR-MDX mutant mice show less severe muscular dystrophy than MDX mice. Neuromuscul. Disord. 8, 542-550.

Mehrke, G., Brinkmeier, H., and Jockusch, H. (1988). The myotonic mouse mutant ADR: electrophysiology of the muscle fiber. Muscle Nerve $11,440-446$.

Ptacek, L. J., Johnson, K. J., and Griggs, R. C. (1993). Genetics and physiology of the myotonic muscle disorders. N. Engl. J. Med. 328, 482-489.

Puljak, L., and Kilic, G. (2006). Emerging roles of chloride channels in human diseases. Biochim. Biophys. Acta 1762, 404-413.

Pusch, M. (2002). Myotonia caused by mutations in the muscle chloride channel gene CLCN1. Hum. Mutat. 19, 4223-4434.

Reininghaus, J., Fuchtbauer, E. M., Bertram, K., and Jockusch, H.
(1988). The myotonic mouse mutant ADR: physiological and histochemical properties of muscle. Muscle Nerve 11, 433-439.

Steinmeyer, K., Ortland, C., and Jentsch, Y. J. (1991a). Primary structure and functional expression of a developmentally regulated skeletal muscle chloride channel. Nature 354, 301-304.

Steinmeyer, K., Klocke, R., Ortland, C., Gronemeier, M., Jockusch, H., Grunder, S., and Jentsch, Y. J. (1991b). Inactivation of muscle chloride channel by transposon insertion in myotonic mice. Nature 354, 304-308.

Stimson, D. (2001). Myotonia congenita: a muscle disease concealed by muscular physique. Quest 8, 28-33.

Stuhlfauth, I., Reininghaus, J., Jockusch, H., and Heizmann, C. W. (1984). Calcium-binding protein, parvalbumin, is reduced in mutant mammalian muscle with abnormal contractile properties. Proc. Natl. Acad. Sci. U.S.A. 81, 4814-4818.

van Lunteren, E., and Moyer, M. (2003). Wheel running exercise alters rat diaphragm action potentials and their regulation by $\mathrm{K}^{+}$channels. $J$. Appl. Physiol. 95, 602-610. van Lunteren, E., Moyer, M., and Pollarine, J. (2004). Reduced amount and disrupted temporal pattern of spontaneous exercise in diabetic rats. Med. Sci. Sports Exerc. 36, 1856-1862.

van Lunteren, E., Moyer, M., and Pollarine, J. (2007a). Genetic CLC-1 chloride channel deficiency modifies diaphragm muscle isometric contractile properties. Respir. Physiol. Neurobiol. 155, 220-226.

van Lunteren, E., Pollarine, J., and Moyer, M. (2007b). Isotonic contractile impairment due to genetic CLC-1 chloride channel deficiency in myotonic mouse diaphragm muscle. Exp. Physiol. 92, 717-729.

van Lunteren, E., Spiegler, S., and Moyer, M. (2011). Fatigue-inducing stimulation resolves myotonia in a drug-induced model. BMC Physiol. 11, 5. doi: 10.1186/1472-679311-5

Watkins, W. J., and Watts, D. C. (1984). Biological features of the new A2Gadr mouse mutant with abnormal muscle function. Lab. Anim. 18, 1-6.

Weinberg, J., Curl, L. A., Kunci, R. W., and McFarland, E. G. (1999). Occult presentation of myotonia congenita in a 15-year-old athlete. Am. J. Sports Med. 27, 529-531.

Conflict of Interest Statement: The authors declare that the research was conducted in the absence of any commercial or financial relationships that could be construed as a potential conflict of interest.

Received: 03 March 2011; accepted: 25 July 2011; published online: 09 August 2011.

Citation: van Lunteren E, Moyer M, Cooperrider J and Pollarine J (2011) Impaired wheel running exercise in CLC-1 chloride channel-deficient myotonic mice. Front. Physio. 2:47. doi: 10.3389/fphys.2011.00047

This article was submitted to Frontiers in Exercise Physiology, a specialty of Frontiers in Physiology.

Copyright (C) 2011 van Lunteren, Moyer, Cooperrider and Pollarine. This is an open-access article subject to a nonexclusive license between the authors and Frontiers Media SA, which permits use, distribution and reproduction in other forums, provided the original authors and source are credited and other Frontiers conditions are complied with. 